RODRIGO VARGAS CALLEGARI

ESCUELA DE DISENO

FACULTAD DE ARQUITECTURA

UNIVERSIDAD DE VALPARAÍSO

VALPARAÍSO, CHILE

RODRIGO.VARGAS@UV.CL

JUAN CARLOS RODRÍGUEZ TORRENT

ESCUELA DE DISENO

FACULTAD DE ARQUITECTURA

UNIVERSIDAD DE VALPARAÍSO

VALPARAÍSO, CHILE

JUAN.RODRIGUEZ@UV.CL
Fecha de recepción: 13/03/2019

Fecha de aceptación: 24/05/2019

Cómo citar: Vargas Callegari, R. y Rodríguez

Torrent, J (2019) Profesionalización del Diseño

en Chile, una sinfonía en cuatro movimientos.

RChD: creación y pensamiento, 4(6), 1-13

DOI: $10.5354 / 0719-837 \times .2019 .53636$

\section{Profesionalización del Diseño en Chile, una sinfonía en cuatro movimientos}

\author{
Professionalization of Design in Chile, \\ a Symphony in Four Movements
}

Resumen. Este artículo se desarrolla en torno al Diseño, y su posicionamiento social en tanto profesión. Se establece que en los campos profesionales existen reglas, poderes, conflictos y escrutinios permanentes y necesarios para la actualización y vigencia de sus postulados. Se presentan hitos relativos a su historia y saber, como intentos por legitimar una posición en el marco de la división social del trabajo. Sostenemos que los centros de enseñanza son intensamente interpelados por estudiantes, empleadores, las comunidades profesionales y la sociedad; es imprescindible conocer cómo ha operado el microsistema del Diseño académico, qué preguntas se hacen las instituciones, y cómo, y desde dónde se las intenta responder. Con ello, la sintonía con los tiempos sociales, la industria manufacturera y las propias expectativas de las nuevas generaciones, demandan el establecimiento de certidumbres sobre el saber exclusivo del Diseño. Metodológicamente, este trabajo se desarrolla a través de entrevistas a un conjunto de actores relevantes, además de revisión de fuentes secundarias, y Teoría Fundamentada. Palabras clave: campo, Diseño, epistemología, habitus, profesión

\footnotetext{
Abstract. This article is developed around Design, and its social positioning as a profession. It is established that in the professional fields there are rules, powers, conflicts and permanent scrutinies necessary for the update and validity of its postulates. Milestones related to its history and knowledge is presented, as attempts to legitimize its position within the framework of the social division of labor. We maintain that the teaching centers are intensely interpellated by students, employers, professional communities and society, being essential to know how the microsystem of academic design has operated, what questions are asked by the institutions, and how, and from where are they attempted? With this, the harmony with working times, the manufacturing industry, and the expectations of the new generations demand the establishment of certainties about Design exclusive knowledge. Methodologically, this work is carried out through interviews with a group of relevant actors and reviews of secondary sources and Grounded Theory.

Keywords: design, epistemology, field, habitus, profession.
} 


\section{La sociedad de los profesionales}

Sinfonía es una composición musical concebida para ser interpretada por una orquesta que consta de tres o cuatro movimientos de larga duración, con unidad de tono y desarrollo (Diccionario Oxford).

Desde el inicio de la modernidad, las profesiones son la forma más institucionalizada del trabajo. Desplazaron a las "ocupaciones liberales" y surgen como forma legitimada de prestar y de acceder a servicios especializados y validados socialmente como indispensables para la vida contemporánea, donde los profesionales son retribuidos en confianza y en términos materiales. Sociológicamente, las profesiones se expresan como mecanismos para comprender las transformaciones de la sociedad industrial, donde la división del trabajo y la ética detrás de la experiencia colectiva, son vistas como estructuradoras de una nueva sociedad (Durkheim,1982).

El estudio crítico de las profesiones explica los microespacios que configuran el mundo de los especialistas, y toma como referentes a Bourdieu (1995; 1997), Foucault (1995), Freidson (1988), Abbott (1988), Rodríguez y Guillén (1992), entre otros. Para este grupo de filósofos y sociólogos, las profesiones son espacios de poder donde se ritualizan prácticas para la reproducción esotérica de comunidades, las que administran un conocimiento específico, abstracto y exclusivo, conforme a la selección, iniciación y formación de sus agentes profesionales. Hipotéticamente, debemos entender que serían los diseñadores los encargados de reproducir una comunidad profesional del Diseño, a través de la transmisión de contenidos y el cultivo de una epistemología propia. Una profesión constituye un campo de poder: con luchas, objetos e intereses específicos, y con protocolos que se traducen en autonomía del saber, ya que aseguran a través del aprendizaje de un conocimiento exclusivo evitar influencias y prácticas heterónomas. Recluta, regula y reproduce su comunidad a través de un código ético, para dialogar con el Estado y la opinión pública. Constituye una unidad social de responsabilidad, y ofrece un marco de representación simbólica para sus profesantes, los que mediante prácticas ritualizadas libran luchas en torno al posicionamiento del saber que producen modos expertos, y formas de comprensión y explicación del mundo que despliegan sus miembros en el mercado del trabajo.

La ritualización de prácticas o habitus profesional condensa esquemas generativos desde los cuales las comunidades perciben el mundo y actúan en él. Establece límites estructurados que, a lo largo de la historia, permiten dialogar con la estructura social y participar de las relaciones sociales. La internalización de parte del mundo desde las experiencias teóricas y prácticas define lenguajes estructurantes que se expresan en pensamientos, percepciones, ideas, explicaciones y acciones que toman la forma de disposiciones verdaderas, que supera teóricamente el determinismo estructural y el subjetivismo. Así, los individuos construyen a las profesiones, y las profesiones definen a los individuos al otorgarles una identidad social.

Entonces, las profesiones son esencialmente construcciones sociales, históricas, territorializadas y desarrolladas por individuos. En ellas, los agentes se desenvuelven y desarrollan a tiempo completo, en virtud de un objeto e interés. El prestigio del campo se asocia a la correlación de fuerza que son capaces de establecer sus miembros, agentes y las instituciones formadoras en 
las que se canaliza el saber. En la lucha por su posicionamiento, la profesión busca siempre alcanzar el monopolio y la autoridad sobre el objeto definido como propio, para conservar, modificar y transferir el conocimiento. De este modo, las profesiones representan mercados para los capitales específicos de sus miembros, donde estudio y trabajo trasladan privilegios materiales y simbólicos desde y hacia el resto de la sociedad, actuando para y por sus recursos; performan a los profesantes de un campo, redistribuyendo el poder devenido de capitales sociales, culturales, materiales y simbólicos.

El mundo profesional establece una diferencia esencial con respecto a los oficios y las ocupaciones que la modernidad se encargó de desplazar y reubicar. Las profesiones invisten de reconocimiento a aquellos que internalizaron a través de elementos de legitimación, como certificaciones y diplomas, el dominio del conocimiento exclusivo.

Por otra parte, el Estado observa y evalúa las acciones desarrolladas por estas comunidades, y cuando considera que su práctica representa un valor para el proyecto social, las protege con normativas ad hoc, y les resguarda mediante leyes de invasiones de otras comunidades profesionales y no profesionales, consagrándoles un espacio exclusivo de desempeño. De esta manera, la profesionalización es un proceso de resultados inciertos en el tiempo. Algunas ocupaciones y prácticas nunca alcanzan la calidad de profesión, ya que depende de la capacidad de acción interna de las comunidades en la definición de límites, de factores ambientales donde se hallan insertas, y la invasión de otras comunidades disciplinares.

Desde su constitución, las variaciones que pueda sufrir el campo profesional son siempre dentro del espacio de poder y legitimidad social e institucional, en un juego tensionado entre la ortodoxia y la doxa. De ahí que, lo que ha dado estabilidad y regularidad a los campos profesionales más paradigmáticos (Medicina, Derecho, Ingeniería) es el esfuerzo continuo por agenciar una función específica puesta en circulación para ser avalada por la sociedad y el Estado. De este modo, el campo profesional es simultáneamente visión de mundo y división del mundo. Es decir, solo considera a quienes hacen suyo el "punto de vista" como una forma de autorización para quienes se integran al campo, y excluye a todos los demás (Medicina frente a Enfermería, Kinesiología, Odontología).

En el campo disciplinar del Diseño nacional son escasos los cuerpos escriturales que remitan a lo que podríamos llamar "memoria del Diseño chileno" como representación de límites y exclusividad. Tenemos cuatro números de

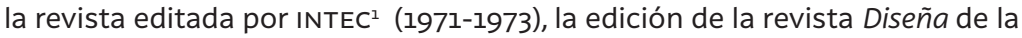
Pontificia Universidad Católica; revista RchD: Creación y Pensamiento, de la Universidad de Chile; revista $180^{\circ}$ de la Universidad Diego Portales, revista Base de la Universidad del Desarrollo; los libros de síntesis de las Bienales; otros intentos editoriales misceláneos como la revista Diseño y Diseño etc. Los artículos y trabajos como los de Hugo Palmarola (2003), los libros publicados por Eduardo Castillo (Castillo, 2010; 2014), el de la socióloga colombiana Eden Medina (2013), y los trabajos de Pedro Álvarez Casselli (2008; 2011). También encontramos, con distintos énfasis, los trabajos de Vico y Osses (2009) y Cristi y Manzi (2017), Malacchini (2015) sobre la identidad y la experiencia de la gráfica y del cartel desde el gobierno de Salvador Allende a la dictadura, revisiones de la transición que hacen los códigos estilísticos y sus mensajes, y cómo éstos van respondiendo a causas tanto internas como externas, y a múltiples dimensiones como la Reforma Agraria, Reforma Universitaria, eventos de coyuntura, conmemoraciones, visitas, agresiones, arte
1. Instituto Tecnológico de la coRfo. 
2. En el volumen $\mathrm{N}^{\circ} 9$ de la Revista Diseña de la

Pontificia Universidad Católica de Chile, al final,

se hace un listado de aproximadamente 100

referencias a publicaciones de Diseño. Sin em-

bargo, su análisis no indica que se trate necesariamente de contribuciones en el campo epistemológico del Diseño, sino que más bien confirma la tesis de este artículo, que el fenómeno de la escritura, la filosofía y el rigor epistemológico es reciente y creciente.

3. En el año 2010 recibieron matrícula un total de 192 programas en la educación terciaria que llevaban el adjetivo "diseño" en su nombre. Fuente: MINEDUC, SIES, consultado en agosto 2016. popular, cambios culturales y económicos, partidos políticos; y más tarde, como respuesta a la violación de derechos humanos, el exilio, la conculcación de las libertades².

Frente a esta producción reciente, es menester preguntarse dónde encontrar lo epistemológico, canónico y recurrente que le da forma a nuestro Diseño como campo, y cómo podría ser transmitido lo substantivo de un conocimiento si no es a través de la escritura.

Las pocas referencias y referentes que prospectan el propio campo epistemológico (ver Bourdieu, 1995; 1997) lo hace incierto para académicos, estudiantes e interesados. Es importante para el Diseño proporcionar una idea sobre dónde y cómo debe ser leído y entendido en términos históricos, a través de sus prácticas, practicantes y productos. Lo anterior fundamenta la traza de su constitución y vigencia, y lo que ha sido filosófica y epistemológicamente permanente en 50 o 60 años desde la fundación de la primera escuela de Diseño moderno.

\section{Consideraciones metodológicas}

Sintéticamente, revisado el acervo bibliográfico referido a Diseño, y en un interés breve de periodización del Diseño en Chile, no aparecen explicitados cuáles son los mecanismos de transformación de la profesión. Ante la falta de documentación académica y gremial que permita establecer una trayectoria profesional, para construir el argumento se debió recurrir a fuentes primarias - mediante entrevistas - para recuperar y establecer una memoria con sus recuerdos y olvidos, y así completar la cadena.

De este modo, se realizaron un número de veintisiete entrevistas, dentro del marco de un proyecto mayor. El universo de la muestra consideró a diseñadores profesionales, directores de Escuela, exdirectores, académicos y pares evaluadores; se incluye a Gui Bonsiepe y Silvia Fernández. La muestra definió un universo en función de criterios como la trayectoria profesional, grados académicos y jerarquías, unidades académicas de universidades del Estado, privadas-tradicionales, privadas e institutos profesionales; las entrevistas se concretaron en Chile, Argentina y México.

En entrevistas, cuya duración fue de entre una hora y treinta y una hora y cuarenta y cinco minutos, las preguntas tuvieron una estructura triádica: a) descriptivas (háblame de...); b) focalización (cuántos tipos existen; cuántos tipos reconoces); c) distinción (cuál es la diferencia entre a, b, c y d). A través de ellas, se buscaba establecer si todos los "casos de Diseño" conocidos, tienen un origen en común, comparten metodologías y técnicas para responder a similares problemas que plantea el proyecto social, es decir, que pudieran ser considerados como una misma profesión. Luego, frente a la existencia de una gran cantidad de programas ${ }^{3}$, nos planteamos responder a partir de la evidencia: ¿cómo se configura históricamente la representación del campo del Diseño?, ¿cómo se explica sistémicamente la gran cantidad de variaciones dentro del campo? y ¿se puede decir que el Diseño se encamina hacia una profesionalización, o se trata de una especie de ocupación polisémica? Diversos entrevistados reconocen esta condición crítica, y necesaria de explorar, ya que a través de la naturalización de una aparente diversidad, se expresan versiones del Diseño que podrían no estar emparentadas, ser carreras diferentes, o pertenecientes a campos epistemológicos distintos. Es decir, los programas, las lógicas de enseñanza y los objetivos podrían ser tan disímiles que resulten irreconocibles bajo una denominación común (Vargas et. al., 2018). 


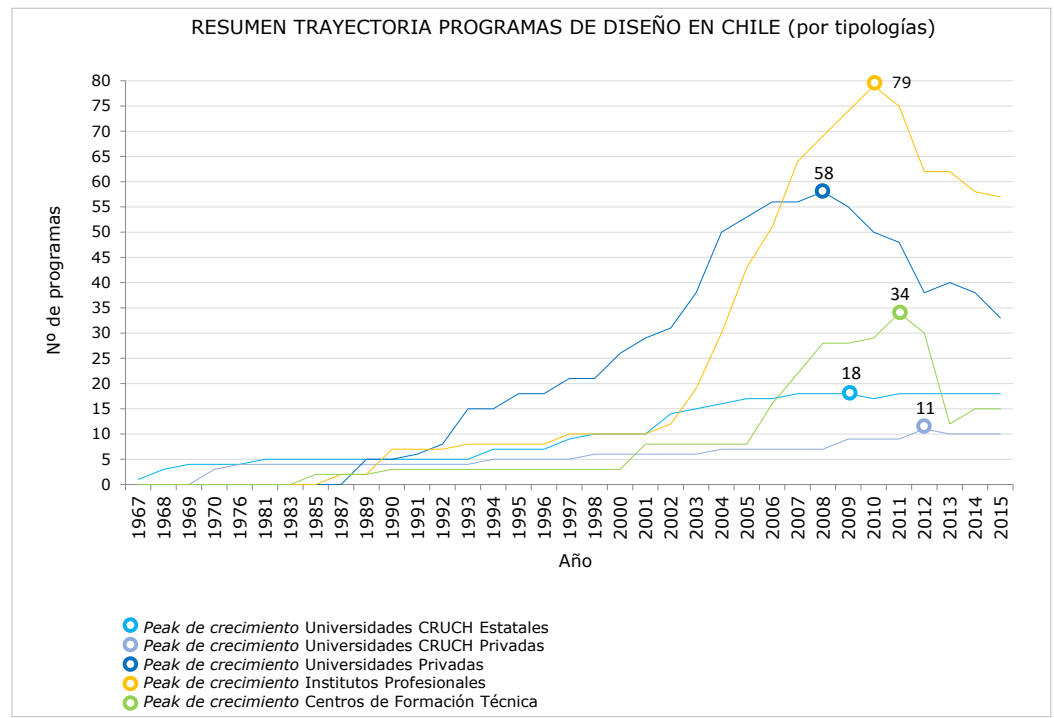

La argumentación se apoyó en un conjunto de exposiciones y manuscritos desarrollados por los autores, tanto en Chile como en el extranjero, recurriendo a fuentes secundarias de información como revistas y publicaciones, así como los programas de formación, los que son tratados como objetos. Para el análisis de la información se utilizó Teoría Fundamentada (Strauss, A. \& Glaser, B., 1967), ya que no depende estrictamente de estudios previos. También, por su flexibilidad para procesar información procedente de fuentes diversas. Sus características como proceso subjetivo se acomodan a una investigación exploratoria, porque permite la emergencia de preguntas e hipótesis para avanzar en el conocimiento profesional del Diseño. El análisis de datos se hace mediante la codificación selectiva, abierta y axial, y posterior proceso de validación. A partir de las entrevistas, identificamos cuatro movimientos como fórmula de configuración del campo, para contribuir con una perspectiva sociológica de la historia del Diseño en Chile.

\section{Historia de una sinfonía}

Al confirmar que las profesiones solo pueden entenderse mediante análisis de tipo histórico, y que los procesos de profesionalización son siempre territorializados, hemos elaborado el siguiente gráfico (ver figura $\mathrm{N}^{\circ} 1$ ) para explicar la deriva histórica de los programas de formación del Diseño en Chile, lo que permite periodizar los momentos de esta "sinfonía en cuatro movimientos". Un programa de formación es una serie de dispositivos humanos y no humanos que comparecen en la transmisión organizada de un conocimiento teórico y práctico, sobre lo que se espera de un profesional del Diseño. Esto implica la declaración de los objetivos, los perfiles de egreso, las mallas curriculares, los cuerpos académicos, la infraestructura y los tipos de organización.

La figura $\mathrm{N}^{\circ} 1$ constata que los programas de formación en Diseño moderno nacen en 1967 en el seno de la universidad estatal. Luego, con cambios sucesivos en la normativa legal, se da paso a la aparición de programas de Diseño en otro tipo de instituciones como institutos profesionales y centros de formación técnica, además de universidades privadas y tradicionales (las
Figura 1. Gráfico que muestra un resumen de la trayectoria de programas de Diseño en Chile, según tipologías. Fuente: elaboración propia con datos de MINEDUC y SIES (agosto 2016) 
4. Fuentes: MINEDUC y SIES, consultadas en agosto 2016.

5. En este punto, es importante señalar que Diseño estaba inscrito en un proyecto más ambicioso de Facultad. Se trataba de un proyecto global de arte y tecnología, ya que trataba entre otras materias, de planificación urbana, desarrollo del cine y la televisión. antiguas privadas). Esta población alcanzó un peak de crecimiento a mediados de la década de 2000 para comenzar su contracción. Sin embargo, no alcanza a detallar las dinámicas políticas, laborales, tradicionales, académicas e ideológicas que han dado forma a lo que actualmente llamamos Diseño.

\section{Primer movimiento}

En $1967^{4}$, en la Universidad de Chile, sede Valparaíso, se crea la primera carrera de Diseño industrial universitaria del país. Los artistas y arquitectos, "sin proponérselo", "casi por casualidad", dan forma a la naciente carrera y la constituyen en un nuevo espacio de desarrollo académico al alero de la Universidad de Chile. Se trataba de una "versión menor" de los dominios de la arquitectura; se perfila como una especie de subocupación disciplinar, o como una versión del "arte más aplicado" y menos voluntarista y subjetivo, "más acorde con los nuevos tiempos", o "un arte más comercial”.

Así, se elaboran los primeros programas para Diseño, con nociones e ideas provenientes de ámbitos tan subjetivos como la experiencia individual, un viaje al extranjero de alguna autoridad administrativa, o lo que se podía recoger de las pocas revistas internacionales que Ilegaban a Chile. Esta situación, mayormente empírica e intuitiva, expresa una cierta insularidad, en la medida que Diseño ya tenía antecedentes suficientes de efectividad y estratégica posición en la estructura productiva de Europa y Japón, con posterioridad a la Segunda Guerra Mundial.

Casi simultáneamente a la creación del primer programa, en octubre de 1968, llega Gui Bonsiepe a Chile. Ganador de un concurso de la Organización Internacional del Trabajo (OIT), fue elegido para desarrollar un proyecto de fomento a la pequeña y mediana empresa basado en Diseño industrial, desde el Servicio de Cooperación Técnica (SERCOTEC), de la Corporación de Fomento de la Producción (CORFo) y el Instituto de Investigación Tecnológica (INTEC). Es decir, un año después, y en paralelo a la apertura del primer curso oficial de formación en Diseño en la Universidad de Chile de Valparaíso, se introduce una versión no académica, inserta directamente en la institucionalidad de gobierno como política pública, como un aliado para el proyecto estatal de substitución de importaciones y de búsqueda de independencia tecnológica. La política pública refiere al conjunto de acciones, medidas, decisiones y estrategias que un estado realiza o decide adoptar, con el claro objetivo de satisfacer un conjunto de necesidades públicas (colectivas) de tipo coyuntural o estructural, para responder a esos objetivos identificados como estratégicos y prioritarios. De este modo, alineado con el mandato de disminuir las brechas con las economías centrales, se trataba de "contribuir a reducir la dependencia tecnológica en la industria manufacturera" (Bonsiepe, 2016, p. 10); enfocándose en siete áreas de problemas: maquinaria agrícola, productos para el consumo básico, equipamiento para el área de salud e instrumental médico, máquinas y herramientas para la industria ligera, componentes para la construcción, y envases para el transporte y consumo. En este caso, los mandantes fueron los ministerios en consecuencia con la reorganización del sistema productivo y un proyecto político.

El trabajo de Bonsiepe al mando del Grupo de Diseño industrial estuvo vinculado a profesionales como Stafford Beer, Fernando Flores, Pedro Domancic, Michael Weiss, Wolfang Eberhagen, Werner Zemp, Guillermo Cintolesi (Medina, 2013); además de cuatro estudiantes de la nueva carrera de Diseño industrial de la Universidad de Chile de Santiago, Guillermo Capdevila, 
Alfonso Gómez, Fernando Shultz y Rodrigo Walker, quienes fueron capaces de desarrollar más de veinte proyectos de trascendencia, con los cuales queda en evidencia el potencial transformador del Diseño para la industria y para la sociedad (véase Palmarola, 2003; Medina, 2013; Bonsiepe, 2016). De lo anterior, se desprende que el Diseño moderno en Chile posee un origen dual. Por un lado, con una versión academicista desarrollada por agentes provenientes de otros campos disciplinares, que ven al Diseño como una oportunidad de extender el poder profesional de arquitectos y artistas; $y$, por otro, una versión estructural-funcionalista incubada por mandato estatal, que opera directamente desde la institucionalidad de Gobierno, en departamentos de investigación y tecnología, para el fomento de la industria y el consumo de bienes internos, y dirigida por un diseñador europeo con experiencia en un proyecto similar en la Alemania de posguerra. Para Eduardo Castillo (2014), el origen del Diseño podría situarse junto a la Escuela de Artes y Oficios (EAO) de 1849. La trayectoria de este proyecto educativo civilizatorio si bien cumplió un papel fundamental en la enseñanza de oficios y un saber industrial, para nosotros este legado indiscutible de la FAO no está suficientemente esclarecido aún para sentar un principio de origen del Diseño. Como sostiene el mismo Castillo, la EAo no alcanzó a conciliar la "separación de mundos entre arte e industria [lo que] restó fuerza al camino del diseño en el medio nacional" (p. 615), y quedó reflejado en la absorción por parte de la Universidad Técnica del Estado de aquellas áreas más técnicas, desentendiéndose de expresiones humanistas del proyecto. Asimismo, los archivos MINEDUC y SIES consideran al primer programa de Diseño en la actual Universidad de Valparaíso, otrora Universidad de Chile, sede Valparaíso.

\section{Segundo movimiento}

El segundo movimiento se encuentra marcado por la lucha por delimitar el campo académico de Diseño y definir de qué se trata el estudio y contenido de la nueva carrera. Los estudiantes del Diseño de la Universidad de Chile de Santiago se encontraron con dificultades para homologar en los circuitos netamente académicos los conocimientos y experiencias acumuladas en el Grupo de Diseño Industrial INTEC, asociados a tecnología y compromiso con el bienestar social. En 1973, en un contexto de Guerra Fría, el Golpe cívico-militar acaba rápidamente con el proceso de inserción profesional de Diseño en el Estado. Bonsiepe es expulsado de Chile y en 1974 se desarticula INTEC. Así termina un único ejemplo a nivel mundial en el cual el Diseño se incorpora como profesión desde arriba (Medina, 2013), ya que lo usual es tener que demostrar el valor de la acción profesional en resultados, de modo de ganarse un espacio protegido para el desempeño de la comunidad.

El Diseño académico se desarrolló a partir de cuatro escuelas universitarias durante la década 1970. La Universidad de Chile sede Valparaíso, Universidad Católica de Valparaíso, Universidad de Chile de Santiago y Universidad Católica de Santiago. De esta época no existe una documentación suficiente para recuperar una trayectoria histórica del campo; más bien, de acuerdo a lo que revelan las entrevistas, se tendió a "mantener lo que hasta entonces había". Una necesaria discusión sobre los fundamentos y objetivos académicos profesionales se truncó, por temor a las condiciones del contexto político autoritario y por el miedo a "perder los trabajos". De hecho, algunas carreras como Sociología, Antropología y Filosofía -según recuerdan Shultz y Walkerfueron suspendidas o intervenidas políticamente.
6. Establece reconocimiento oficial a aquellas instituciones creadas por la citada ley, a las que se les concede una autorización de funcionamiento, y a las que se crean a partir de la Ley Orgánica Constitucional de Enseñanza. De este modo, se legitima a todas las existentes al 31 de diciembre de 1980 (U. de Chile, Universidad de Santiago -otrora Técnica del Estado-, Pontificia Universidad Católica de Chile, Universidad de Concepción, Universidad Federico Santa María, Pontificia Universidad Católica de Valparaíso, Universidad Austral de Chile y Universidad Católica del Norte -otrora U. del Norte-), y las que se derivan o son sucesoras de ellas, las que mantendrán plena autonomía para otorgar títulos profesionales y grados académicos.

6. Entre los elementos de distinción de instituciones terciarias de educación en Chile, en términos formales, está la fuerte tradición humanista de los estudios universitarios en contraposición a la oferta técnica y técnico-profesional. Sin embargo, para el caso de las carreras de Diseño y sus egresados, el mercado del trabajo escasamente puede establecer diferencias notables entre estos niveles, salvo con las universidades de excelencia. 
6. Establece reconocimiento oficial a aquellas instituciones creadas por la citada ley, a las que se les concede una autorización de funcionamiento, y a las que se crean a partir de la Ley Orgánica Constitucional de Enseñanza. De este modo, se legitima a todas las existentes al 31 de diciembre de 1980 (U. de Chile, Universidad de Santiago -otrora Técnica del Estado-, Pontificia Universidad Católica de Chile, Universidad de Concepción, Universidad Federico Santa María, Pontificia Universidad Católica de Valparaíso, Universidad Austral de Chile y Universidad Católica del Norte -otrora U. del Norte-), y las que se derivan o son sucesoras de ellas, las que mantendrán plena autonomía para otorgar títulos profesionales y grados académicos.

7. Entre los elementos de distinción de instituciones terciarias de educación en Chile, en términos formales, está la fuerte tradición humanista de los estudios universitarios en contraposición a la oferta técnica y técnico-profesional. Sin embargo, para el caso de las carreras de Diseño y sus egresados, el mercado del trabajo escasamente puede establecer diferencias notables entre estos niveles, salvo con las universidades de excelencia.

8. Cabe señalar que desde la década de 1960 existieron instituciones como el Instituto de Ciencias y Artes (INCA) y el Centro de Estudios Aplicados (CEA). 9. Coloquialmente aquí aparecerían en el sistema de educación superior, y en Diseño, lo que se conoce como "carreras de tiza y pizarrón". 10. Con la idea de "programas pesados", nos referimos a que había cuestiones curriculares propias o muy cercanas a las ingenierías. Entre estas: cálculo, física, química, resistencia de materiales. Aún no se ha explorado si este cambio ha tenido que ver con la feminización de la matrícula en Diseño. 11. En la década de 1980 surgen intentos por agruparse gremialmente, aunque sin alcanzar niveles de representatividad importantes debido a disputas internas y falta de acuerdos respecto de la orientación que debía tener un Colegio Profesional, y quiénes deberían dirigirlo, cuáles serían sus motivaciones y funciones. Esta situación no hace más que ilustrar profundas diferencias epistémicas sobre la profesión y la lucha de poderes entre la institucionalidad académica y el poder profesional alcanzado por algunos de sus agentes más importantes.
En 1981, en plena dictadura, el DFL N ${ }^{\circ} 1$ del 30 de diciembre de 1980 fomenta la creación de instituciones de educación superior de carácter técnico-profesional ${ }^{6}$ y de régimen de las doce carreras que tendrán el estatus de universitarias, entre las que no está Diseño. La idea de esta reforma fue desarrollar un sistema de varios niveles para alcanzar la "masificación" de la educación superior. A partir de ese momento, a las universidades, depositarias de una larga tradición en educación superior que contempla además de la docencia, la investigación, la creación y la extensión, se suman los Institutos Profesionales (IP) y Centros de Formación Técnica (CFT) ${ }^{7}$, esencialmente para la realización de docencia, como oferta en educación terciaria ${ }^{8}$. Con la irrupción de nuevos niveles y centros de educación superior, la comunidad de diseñadores frente a una industria que desaparecía ante las importaciones observa que la docencia en Diseño puede ser una vía alternativa al desarrollo profesional, y surge el Diseño academicista como una oportunidad laboral para diseñadores egresados de algunos de los programas existentes hasta 1980. También comienza una ramificación de la enseñanza de Diseño hacia la educación técnica y técnico-profesional, lo que marca el comienzo de la expansión acelerada de Diseño como especie (véase Figura $\mathrm{N}^{\circ} \mathrm{l}$ ).

La expansión del Diseño hacia campos técnicos y técnico profesionales, mediante la implementación de programas curriculares de corta duración, hace circular nuevas versiones en comparación con los programas universitarios existentes. Esta reducción curricular de Diseño evidentemente actúa como mecanismo selector de lo que sería "apropiado enseñar del Diseño" y de cuáles serían los mínimos propios del campo`. Aparecen versiones basadas en programas desprendidos de filosofía, economía, sociología, matemáticas, poesía, física y otras especialidades, que aparecían en algunos de los referentes universitarios de la profesión, con el fin de "alivianar esos currículos tan pesados de Diseño en las universidades" ${ }^{\prime 10}$.

Los nuevos programas producen un profesional distinto, que paulatinamente va -a través de este "adelgazamiento curricular"- perdiendo conexión con un tipo de industrias productivas y con el horizonte laboral que había prefijado en INTEC. Con un currículum más lábil, disperso, acotado y menos ortodoxo, de acuerdo a la experiencia europea, se produce la enunciación y circulación de nuevos discursos desde lo académico hacia la práctica, que promueven una diferenciación interna en el campo académico.

De este modo, en la construcción de estas identidades hay vacíos epistemológicos sobre los objetivos y el saber exclusivo que nunca se llenan. En los intramuros académicos emerge un enjambre de voces politonales de un coro desafinado a falta de una continuidad semántica, de una crítica especializada, de circuitos de referencia, representaciones autorizadas, una voz legitimada desde lo gremial ${ }^{11}$, una producción académica teórica o histórica capaz de fijar ciertos hitos, y acuerdos básicos que permitieran revisar y discutir el rumbo de la profesión. Se produce una reinvención del Diseño al alero del crecimiento del mercado de la educación en paralelo a la transformación del modelo económico. Cada unidad académica, según su parecer y sorteando luchas internas, fija sus propios objetivos para el Diseño; y cada académico enseña conforme a su aprendizaje previo, echando mano a sus propios capitales, intereses e intuiciones. El corolario es un débil espesor epistemológico para el intento de profesionalización. 


\begin{tabular}{|c|c|c|c|c|c|c|c|c|c|c|}
\hline Origen institucional / años & 2007 & 2008 & 2009 & 2010 & 2011 & 2012 & 2013 & 2014 & 2015 & 2016 \\
\hline Consejo de rectores (CRUCH) & 3461 & 3440 & 3292 & 3322 & 3186 & 3062 & 2975 & 2855 & 2787 & 2752 \\
\hline Privadas tradicionales & 1070 & 1176 & 1322 & 1400 & 1444 & 1428 & 1425 & 1522 & 1575 & 1650 \\
\hline Privadas & 4677 & 4449 & 4742 & 4787 & 4745 & 4598 & 4321 & 3973 & 3655 & 3338 \\
\hline Institutos profesionales & 9602 & 10095 & 11644 & 13120 & 13311 & 12740 & 11972 & 11152 & 10674 & 10171 \\
\hline Centros de formación técnica & 704 & 1084 & 1226 & 1425 & 1374 & 1214 & 1020 & 947 & 890 & 828 \\
\hline TOTALES & 19514 & 20244 & 22226 & 24054 & 24060 & 23042 & 21713 & 20449 & 19581 & 18739 \\
\hline
\end{tabular}

\section{Tercer movimiento}

En 1990, con el comienzo de una década, también se retoma la democracia como sistema político. Sin embargo, las tres últimas normativas impuestas por la dictadura fueron sobre educación, específicamente abriendo la posibilidad de que actores privados pudieran ingresar al sistema de educación superior con ofertas de carreras y planteles, pero esta vez con mayor autonomía ${ }^{12}$. Comienza un incremento sostenido en la cantidad de programas de formación en Diseño como el expresado en la figura $\mathrm{N}^{\circ} 1$. No solamente en la educación técnica y profesional, sino que también en universidades de origen netamente privado, lo que tiene un fuerte impacto en el número de alumnos matriculados en Diseño (ver figura $\mathrm{N}^{\circ} 2$ ). En lo esencial, tenemos un crecimiento de la población de programas de Diseño, sin discusiones epistemológicas ni de contexto productivo, económico, social, cultural y científico-tecnológico, sino más bien como oportunidades de mercado.

En estos términos, las definiciones del Diseño están dadas por intentos de recomponer el sistema universitario en las nuevas condiciones, mientras se internacionaliza la economía, y se consolida el sistema neoliberal en Chile. Es decir, en lo doméstico se desatendió el desarrollo disciplinar por la protección de los espacios de trabajo, mientras la problemática de los proyectos de Diseño en el plano internacional avanzaba en sincronía con valores de posindustria (Inglehart, 2000).

Pasadas dos décadas, bajo estas "condiciones ambientales", tenemos que, de los aproximadamente 18.700 estudiantes de Diseño del sistema de educación superior, en 2016, solo unos 7.700 pertenecen al sistema universitario, y más de 10.000 al sistema técnico profesional. ¿Quién podría responder la pregunta sobre cómo sucedió este enroque?, ¿cómo Diseño desde su origen en las aulas de la Universidad de Chile se transformó en una carrera técnica?, ¿cuáles fueron los acuerdos profesionales de la comunidad para que esto fuese así?, ¿o solo sucedió como resultado de los acomodos de la industria de la educación?, ¿o individualismo?, ¿falta de cohesión gremial?, ¿acaparamiento de oportunidades?, ¿ausencia de identificación de "necesidades país"?, ¿falta de supervisión y sincronía entre la academia y la sociedad, entre la academia y la industria? La oferta de los nuevos actores y una excesiva dispersión de versiones del Diseño omitió una necesaria reflexión sobre los impactos futuros de una hiperpoblación de diseñadores, ya que algunos carecen de espacios para desempeñarse en el mercado académico y profesional, o se encuentran subocupados. La "omisión" fue anticipada por Bonsiepe hace 50 años en el Manual de Diseño industrial (1969), donde se asegura que el número de diseñadores egresados y titulados adecuados es el número de profesionales que pueda absorber la industria ${ }^{13}$. En 1974, ya fuera de Chile, también advertía sobre los riesgos de llamar diseñador al "estilista de los cabellos" o
Figura 2. Número de estudiantes de Diseño por tipo de institución y crecimiento de la matrícula en CFT, IP y universidades entre 2007-2016. Fuentes: MINEDUC, SIES, consultados en agosto 2016. Elaboración de los autores.

12. Al año 2011, existían sesenta universidades en el país.

13. Señala que, hacia mediados de la década de 1960, en Alemania se titulaban cincuenta y cinco diseñadores por año, lo que era una cifra considerada "alta". Sin embargo, se justificaba bajo el esquema de un país en reconstrucción luego de la Segunda Guerra Mundial, y frente a la necesidad urgente por reindustrializar. 
al "manicurista, designer de las uñas" (Chiapponi, 1999, p. 19), cuyo efecto de ampliar indiscriminadamente los postulados del Diseño a cualquier actividad de la vida humana, terminaría con un profesional vaciado de sus contenidos epistémicos, y el término diseño (en minúscula) resultaría en una técnica o método menor, adaptable a cualquier contexto o situación.

¿Cuántos de los diseñadores, docentes o estudiantes de Chile han estudiado el Manual de Diseño industrial entregado por Gui Bonsiepe en 1969 para dar forma a la actividad en el país?, zacaso Bonsiepe puede no ser un referente suficientemente validado para el Diseño nacional?, ¿de dónde vinieron las nociones de Diseño que dieron forma a esta nueva ola de diseñadores, como cita Chiapponi?, ¿dónde se ubica el mayor grado de expertisse profesional de los diseñadores formados en Chile?, ¿el mundo del trabajo es capaz de reconocer, valorar e integrar a este profesional?

El tamaño de la especie quedó fuera de control. El contexto permitió la vulgarización del Diseño, e incluso lo fomentó, y la comunidad disciplinar no presentó objeciones a la diversificación y multiplicación de la oferta. Primero, porque cada Escuela se encargó de desarrollar formas de protección, y sus académicos se ocuparon de clausurar sus fronteras y conexiones con el exterior, para volcarse por completo hacia lo interno. Segundo, porque la expansión de ofertas de programas de Diseño era también una posibilidad laboral para que aquellos egresados sin redes, ni articulaciones con el Estado o la industria pudieran instalarse desde lo académico y administrativo en otras instituciones. Así, se formaron diseñadores para el aula que reprodujeron acríticamente lo aprendido, produciéndose un desacoplamiento con el desarrollo científico-tecnológico, y cambios sociales y culturales.

\section{Cuarto movimiento}

En la transición al nuevo siglo, el Diseño ya no fue desarrollado principalmente por arquitectos y artistas. Son los diseñadores quienes comienzan a dar forma al campo, ya sea porque vieron la oportunidad que ofrecía el mercado para avanzar hacia un nuevo espacio laboral en la academia o mejorar las oportunidades existentes. En 1990 había más demanda que oferta de profesores para ocupar puestos en diferentes instituciones, y no era extraño encontrar casos donde "profesores de Diseño podrían tener contratos en varias casas de estudios", el duplicar y hasta triplicar las jornadas laborales contratadas. En paralelo, algunos egresados jóvenes comienzan a salir de Chile para continuar estudios de perfeccionamiento y profundización, principalmente a través del grado de magíster. Hacia la última década del siglo XX y comienzos del XXI, se incrementa el número de egresados que deciden ir por la vía académica y continúan estudios de Doctorado en Diseño en el exterior, principalmente en España, Italia e Inglaterra.

Ya de regreso, la presencia de esta nueva clase académica plantea nuevas dificultades para la configuración y estabilización del campo, aunque claramente proponiendo una renovación en los siguientes términos:

1) El reconocimiento de la labor investigativa formal, totalmente desconocida en el mundo de Diseño académico por casi cuarenta años en Chile, junto a la posibilidad de generar conocimiento nuevo;

2) la provisión de espacios internos en las organizaciones donde acoger a este nuevo académico, con su rol como docente e investigador;

3) la necesidad de crear canales donde volcar los resultados de investigación: revistas, seminarios, congresos, encuentros y proyectos; 
4) integrar a la cultura de las instituciones un conocimiento más universal, menos autorreferente y reproductor en sus modos de aprendizaje y trasmisión, con mayor sustento epistemológico y con el traslado de preguntas vigentes en otras latitudes; $y$,

5) la generación de conocimientos y metodologías necesarias para comenzar a producir procesos de acumulación en el campo. En este sentido, la integración del diseñador especialista, del diseñador intelectual o científico, capaz de movilizar temas particulares y agenda propia, irrita a algunas organizaciones, las que ven amenazada la quietud del saber hacer "más cómodo y tradicional", o el "como siempre se ha hecho".

Esta nueva clase de diseñadores produce procesos de diferenciación interna, los que afectan a las formas organizacionales, y se fundamenta en certificaciones e indicadores cienciométricos. Pese a las irritaciones, el paradigma del investigador representa una tendencia que se instala junto con el concepto de Calidad en Educación. El trabajo de investigación para Diseño ha permitido recuperar la memoria temprana del Diseño, en una versión actualizada, crítica y más transdisciplinar. Existen diseñadores-historiadores que han recuperado una historia olvidada, pero también están trabajando profesionales de Diseño formados en otras áreas como la metodología (Briede \& Mora, 2013; Briede \& Rebolledo, 2013), la educación y sociedad (Bravo, 2016), y la profesión (Vargas, 2017), por mencionar algunos. Este cuarto movimiento revisita críticamente las bases de la disciplina desde un trabajo de rescate de referentes y referencias, pero también de creación e innovación de los postulados de Diseño a las que sucumbió el profesional del tercer movimiento en esta sinfonía, aquel que le ha costado encontrar su tono dentro de la armonía. Con estos nuevos agentes se forma una primera "Escuela revisionista" de Diseño en Chile, ya que se recupera la doble historia y se comienza a ir en busca del sentido que puede dar forma a una comunidad epistémica para construir su habitus específico. En lo esencial, se incorporan nuevos procesos de construcción del conocimiento: histórico, metodológico, inter y transdisciplinario, complejo, colaborativo y transparente.

\section{Conclusiones}

Decirse diseñador por tener una certificación de una institución instalada en el campo no es equivalente a tener una posición social y de mercado.

El crecimiento de la plantilla de profesores es un tema en sí mismo en la profesionalización; y el número de estudiantes, otro. El segundo grupo determina la existencia o vulnerabilidad del primero, pero ambos se encuentran en una relación de mercado, y este es implacable.

Academia y profesión: ¿son exactamente lo mismo hoy? La respuesta preliminar es no, especialmente cuando el espesor de los programas se ha adelgazado y la complejidad social y tecnológica va en aumento. Los tiempos teóricos parecen no corresponderse con los tiempos de los fenómenos. La academia ha dado origen a un especialista: el homo academicus. Lo importante para reconocer el lugar y el valor de su habitus como académico, es poder identificar ¿con quién está conectado?, ¿con qué mundos se vincula?, ¿con qué objetivos?, ¿qué lectura hace de la realidad social, cultural, política, económica y ambiental de Chile?, ¿cuál es la contemporaneidad de su discurso? Como en toda profesión, se exige que el discurso pueda ser devuelto a la sociedad y ser sancionado como válido. 
Los cuatro movimientos que pueden ser analizados como proceso de profesionalización del Diseño, finalmente deben esclarecer el horizonte actual del campo. El mercado es demasiado severo según la lógica evolutiva, si lo hacemos equivalente a un ecosistema; $y$, dentro del ecosistema del Diseño, posiciones institucionales, epistemológicas, identitarias y territoriales demasiado débiles, pueden ser absorbidas por artistas, ingenieros, arquitectos, periodistas, psicólogos u otros campos profesionales más consolidados. Los cambios en el ambiente favorecieron -desde la década de 1980- la aparición de toda clase de programas de formación en Diseño y algunos difícilmente pueden justificar su existencia sin diálogo con la nueva industria y sociedad. La densidad de la población de programas de Diseño se ajustará a un tamaño que pueda sostener el poder profesional que la comunidad sea capaz de producir. Las escuelas más fuertes estarán en condiciones de organizar las acciones, delimitar el campo, elaborar los discursos, desarrollar los canales de comunicación, implementar los ritos, establecer los núcleos de saber específico y las redes de conocimiento. Entonces: ¿debemos entender que ese será el discurso oficial del Diseño?, ¿qué les queda a las otras escuelas?, ¿en torno a qué se diferenciarán para sobrevivir en un proceso inevitablemente darwiniano? Al enunciar que el Diseño de la década de 1970 fue un modelo ejemplar y único porque se desarrolló dentro de un proyecto político que le dio sentido, no decimos que hoy Diseño se halle ajeno a un proyecto político. Sostenemos que lo que se encuentra en tensión es la capacidad de las organizaciones por entender su contexto y posición, y junto a ello asumir la responsabilidad de decidir el lugar de la profesión respecto a la concepción estatal y del poder profesional que sus agentes son capaces de portar de acuerdo a ello. Está comenzando un quinto movimiento, en el cual las instituciones mejor preparadas, estables, fuertes y conectadas consolidarán los mejores equipos académicos y programas; para capturar a los estudiantes con mejores capitales, acaparar los recursos más valiosos del sistema y responder de mejor manera a los inputs que entrega el medio. Luego, vendrán las demás organizaciones; se distinguirán las que sean capaces de impregnar coherencia a sus programas, en términos de oferta y posibilidades de llevar a cabo alguna propuesta académica de nicho, y finalmente las demás. Tal como lo dicta el mercado, el sistema se fragmentará entre productos y consumo, subsistencia y mortalidad. 


\section{Bibliografía}

Abbott, A. (1988). The system of professions: an essay on the division of expert labor. Chicago: University of Chicago Press.

Álvarez, P. (2008). Chile Marca Registrada. Santiago: Ocho Libros Editores.

Álvarez, P. (2011). Mecánica doméstica. Publicidad, modernización de la mujery tecnología para el hogar 1945-1970. Santiago: Ediciones Pontificia Universidad Católica de Chile.

Bonsiepe, G. (1978). Diseño industrial, tecnología y dependencia. México: Editorial Edicol.

Bonsiepe, G. (1969). Manual de diseño. Santiago: Organización Internacional del Trabajo (OIT-ONU).

Bonsiepe, G. (2016). Del archipiélago de proyectos, diseño industrial en Chile (1971-1973). La Plata: Nodal.

Bourdieu, P. (1995). Las reglas del arte. Génesis y estructura del campo. Barcelona: Anagrama.

Bourdieu, P. (1997). Capital cultural, escuela y espacio social. Buenos Aires: Siglo XXI.

Bravo, U. (2016). "Diseño para el desarrollo. Vigencia de los principios de Ulm frente a los desafíos del proyecto país". RChD: creación y pensamiento, I (1), pp. 79-93.

Briede, J., \& Mora, M. (2013). Propuesta evaluativa para el Taller de Diseño Centrado en el Usuario (DCU), en la Carrera de Diseño Industrial de la Universidad del Bío-Bío, Chile. Formación Universitaria, 6 (2), 33-42.

Briede, J., \& Rebolledo, A. (2013). "Modelo visual para el mapeo y análisis de referentes morfológicos: aplicación educativa en el diseño industrial, Ingeniare". Revista chilena de ingeniería, vol. $21 \mathrm{~N}^{\circ}$ 2, 2013, pp. 185-195

Castillo, E. (Eds.). (2010). Artesanos, artistas, artífices. La Escuela de Artes Aplicadas de la Universidad de Chile 1928-1968. Santiago: Ocho Libros Editores.

Castillo, E. (2014). La escuela de artes y oficios. Santiago: Ocho Libros Editores.

Chiapponi, M. (1999). Cultura social del producto: nuevas fronteras para el diseño industrial. Buenos Aires: Ediciones Infinito.
Durkheim, E. (1982). La división del trabajo social. Madrid: Akal Ediciones.

Foucault, M. (1995). Las palabras y las cosas. México: Siglo XXI.

Freidson, E. (1988) Professional powers: A study of the institutionalization of formal knowledge. Chicago: University of Chicago Press.

Inglehart, R. (2000). Modernización y posmodernización. El cambio cultural, económico y político en 43 sociedades. Madrid: CIS-Siglo XXI.

Rodríguez, J. y Guillén, M. (1992). "El sistema de las profesiones: el caso de las profesiones económicas en España". Revista Española de Investigaciones Sociológicas, 59/92, pág. 243-259.

Rodríguez, J., Vargas, R. y Molina, R. (2017). "Responsabilidades en el diseño chileno. Reflexiones sobre un campo desbordado". En El Diseño en el fortalecimiento y la integración del desarrollo regional, Actas $3^{\circ}$ Congreso Latinoamericano de Diseño, $1^{\text {a }}$ edición mejorada, pp. 505-513.

Medina, E. (2013). Revolucionarios Cibernéticos, tecnología y política en el Chile de Allende. Santiago: LOM.

Palmarola, H. (2003). "Productos y socialismo: diseño industrial estatal en Chile, en 1973". En La vida cotidiana de un año crucial, pp. 225-295. Santiago: Editorial Planeta.

Glaser, B. \& Strauss, A. (1967). The discovery of grounded theory. Chicago: Aldine Press.

Vargas, R., Rodríguez, J. y Arias, P. (2018). "Diseño, sobrediseño y comunidad epistémica. Una discusión sobre los límites del campo profesional". Revista Base, Volumen 4, $\mathrm{N}^{\circ} 3$, pp. 268-277.

Vargas, R. (2017). "Diseño, una palabra problemática para el posicionamiento profesional". En El Diseño en el fortalecimiento y la integración del desarrollo regional, Actas $3^{\circ}$ Congreso Latinoamericano de Diseño, $1^{a}$ edición mejorada, pp. 13-21. 